\title{
Römische Urteile über Luther und Erasmus im Jahre 1521
}

\author{
nach $\mathrm{J}$ ak ob Zi e gler. \\ Von P. Kalkof.
}

Bei meinen Untersuchungen uber "Lathers römischen Proze日" (ZKG XXV) stieß ich auf eine Notiz Carl Kraffts, der aus einem Briefe des bekannten Theologen und Mathematikers Jakob Ziegler (geb. etwa 1470 zu Landan in Niederbaiern, † 1549 in Passau) an Erasmus mitteilt, daB im Jahre 1521 dreihondert Christen in Rom das Abendmahl unter beiderlei Gestalt gefeiert hätten. ${ }^{1}$ )

Ein solcher Vorgang erschien mir sofort ganz unglanblich; mit der geistigen Verfassung der römischen Bevölkerung, die wohl erst durch die pomphafte Bucherverbrennung am 12. Juni $1521^{2}$ ) und die Predigt des Dominikaners Cyprian Beneti etwas von Luther erfahren haben durfte, wie mit der Haltung der weiteren kurialen Kreise, auch der Landsleute Luthers ist er ganz unvereinbar und demnach auch mit der Glanbwurdigkeit des scharf beobachtenden Berichterstatters unverträglich.

Das Rätsel löste sich beim ersten Blick in das von

1) Aufzeichnnngen des schweizerischen Reformators Heinr. Bullinger über sein Studium zu Emmerich u. Köln (1516-1522), Elberfeld 1870, S. 66: ngerade zu der Zeit, in der sich bei B. in der deutschen Metropole des römischen Katholizismus evangelische Überzeugungen bildeten, sei auch in Rom selbst eine merkw ürdige evangelische Regung hervorgetreten"; Ziegler melde aus Rom: ${ }^{\text {h }}$ ier haben

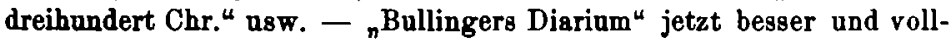
ständig in den Qu. z. schweiz. Ref.-G. hrsg. v. E. Egli, Basel 1904.

9) ZKG. XXV, S. 578 Anm 2.

Archiv fur Reformationsgeschichte III. 1. 
C. Krafit selbst anrezogente Uriginal dieses Briefes in der Rhedigerana hiesiger Stadtbibliothek: da nun die Leydener Ausgabe von diesem sichreiben. vermutlich nach einer recht guten Abschrift. nur drei Abschnitte mitteilt, in denen Ziegler Ratschläge erteilt zur Beschaffung einer im Besitz des Breslauer Domkapitels befindlichen Cyprian-Handschrift und zweier in bairischen Bibliotheken befindlichen kleineren Schriften des Hieronymas, ${ }^{1}$ ) während gerade der grobe Rest durch seine Mitteilungen uber den Lebensgang des Verfassers, das Auftreten Dr. Ecks and Stunicas in Rom uberaus wertvoll ist, so schien es erwunseht. auch diesen Teil baldmöglichst allgemein zugänglich zu machen.

Der Lebensgang des uberaus vielseitigen Forschers, der sich dureh astronomisch-mathematische und geographische wie durch theologische Arbeiten, teils exegetischer, teils apologetischer und polemischer Natur ausgezeichnet hat, verdient uberdies noch die sorgfältigste Berücksichtigung wegen seiner historischen Schriften, die ihn durchweg als einen aufmerksamen Beobachter und unbestechlichen Richter zeigen, der durch die epigrammatische Schärfe seines Urteils, wie sie etwa in seiner bekannten vita Clementis VU. hervortritt, vielfach zu mißtrauischem Widersprach gereizt hat. Doch durfte gerade in diesem Falle bei der Schilderung des vielleicht verderblichsten Pontifikats und der höchst bedenklichen Charaktere in der nächsten Umgebung des Papstes die sittliche Entrustang, die aus dem dentschen Humanisten spricht,

1) Des. Erasmi Rot. opp. omnia, ed. Clericus; tom III, pars II (Lugduni 1703), ep. CCCXX, col. 1699 sq. Ex litteris Jacobi Ziegleri, Landavi Barari. Von unerbeblichen Varianten abgesehen, lies col. 1699 F superioribus vestibus Cyprianum exutum und germanum pondas; die Vermutung einer Lïcke in col. $1700 \mathrm{~B}$ ist hinfällig. Doch ist am Schlusse des Abschnittes iiber Cyprian vor Descripsi olim Ratisponti der c. $1700 \mathrm{~A}$ durch .... angedeutete Satz ausgefallen: Operae precium foeceris, Erasme optime, si tua et Frobenii nostri cura duceres vulgandum, quippiam cui ad cumulum deesse sentires. Hic desiderium tuum ab epistola tua publica, [si] multo ante studiosis declarares, reperires spero ab inexplorato angulo prodeuntem candidum aliquem, qui vos levaret. - Ïber seine textkritischen Bemühungen um Cyprian berichtet Er. am 7 . August 1519 an Spalatin (col. 483 B Fdimus Csprianum, emendatum non mediocri sudore etc. 
suehr berechtigt gewesen sein, und es könnte sich in Einzelfalle nur die Frage erheben, ob Ziegler, dessen Wahrheitsliiebe durch sein ganzes wissenschaftliches Lebenswerk bezeugt wird, in der Lage war selbst und aus naichster Nähe zu breobachten. Da ist es denn von großem Wert. daß durch unsere Quelle seine Anwesenheit in Rom für fast ein ganzes Jahr fruher za belegen ist als bisher, und dab er auch die Regierung Leos $X$. noch in der bevorzugten Stellung eines vom Papste selbst berufenen und in den höchsten Kreisen der Kurie verkehrenden Gelehrten kennen lernte.

Die vortrefflichen Untersuchungen uber Zieglers Leben und schriftstellerisches Wirken, die wir von S. Gunther, Lauchert and besonders von Th. Kolde besitzen, ${ }^{1}$ ) geben für den Anfang seines römischen Aufenthalts kein anderes Zeugnis, als das in der Leydensis enthaltene Datum unseres Briefes; das Bruchstick gestattet jedoch keinen Riickschluß auf die Zeit seines Eintreffens. Doch hätte diese schon weiter zurlickgeschoben werden können durch einige Nachrichten uber die Beziehungen Zieglers zu dem berühmten St. Galler Gelehrten Joachim von Watt, zu dessen jugendlichem Bruder Melchior, der sich in Rom auf die juristische Praxis an der Kurie vorbereitete, Ziegler im Verhältnis eines väterlich wohlwollenden Freandes stand. Er berichtet am 1. August 1526 ans Ferrara an Vadianus, er habe ihm schon 1521 geschrieben and den Brief seinem Bruder mitgegeben, der damals, mit den römischen Verhältnissen unzufrieden, sich im Hause Zieglers zar Ruckreise in die Heimat gertistet habe, aber noch innerhalb der Tore Roms am 20. November erkrankte und am 24. schon im Hospital Santo Spirito in Sassia starb. ${ }^{2}$ )

1) Die Ergebnisse Günthers über Z. als , (ieographen und Jathematiker ${ }^{\mu}$ in $v$. Reinhardstöttners Forschungen z. Kultur- u. Lit.-G. Bayerns IV u. V. hat er selbst, schon ergänzt durch Koldes Beiträge, in der ADB. zusammengefaßt, wo Lauchert über die theologischliterarische Hinterlasseuschaft Z's. berichtet. - Der hier erst später erwähnte Adoptivbruder Zieglers hatte ihn schon 1521 nach Rom begleitet. Er. opp. III, col. $738 \mathrm{E}$.

7) Mitteil. z. vaterl. Gesch. hrsg. v. histor. Ver. in St. Gallen. Tadianische Briefsammiung hrsg. v. E. Arbenz. Bd. XXV (1894), S. 403, 422 f. XXTII (1902), S. 35. In Ferrara weilte Z. wiederholt 
Nach unserem Briefe aber ist Ziegler schon a m 1. März 1521 in Rom eingetroffen, aus Ungarn herberufen rom Papste selbst auf die Empfeblung des Kardinals Hippolit von Este, der als Erzbischof ron Gran, dann ron Erlau (Agriensis) und lebenslänglicher Legat für Ungarn schon durch seine Pfrundenstreitigkeiten eine wichtige Rolle in diesem Königreiche gespielt hat. Indessen dieser Gönner war schon am 1. September 1520 in Ferrara gestorben; ${ }^{1}$ ) dab der Papst dennoch an der Berufung des Deutschen festhielt, erklärt sich nun hinlänglich aus den von Leo $X$. auf den V. Laterankonzil mit Eifer und Verständnis geförderten Bemühungen am eine wesentlich die richtige Berechnung des Osterfestes anstrebende Kalenderreform, die in der dazu bestellten römischen Kommission besonders von dem Niederländer Paul von Middelburg, früher Professor der Mathematik in Padua, jetzt Bischof von Fossombrone ( $\dagger$ in Rom 1534) bearbeitet wurde. ${ }^{2}$ ) (̈̈ber denselben Gegenstand aber hat auch Ziegler eine schrift verfaBt, ${ }^{3}$ ) die wir also auf die Anregang Leos X. zuruickfubren durfen. Der Kardinal ron Este aber hatte Zieglers Befähigung 7u derartigen Arbeiten schon in Ungarn kennen und schätzen gelernt.

Er fuhrte sich beim Papste ein durch Überreichung seiner Schrift „gegen die ketzerischen Pikarden“, d. h. gegen die während eines dreijährigen Aufenthalts im innersten Winkel Mährens (1508-1511) von ihm eingehend beobachteten Brudergemeinden; $;$ wie aus unserem Schreiben hervorgeht, war Ziegler jetzt doch schon von jener scharfen Anfeindang der böhmischen Brüder zurückgekommen; er entschuldigt die

und längere Zeit bei seinem treuen Freunde Celio Calcagnini; die Anknüpfung dieser Beziehungen aber geht also zurïck auf die ron $Z$. schon in Ungarn erworbene Gönnerschaft des estensischen Kirchenfürsten.

1) Marino Sanuto, Diarii XXIX, col. 15̃6. 192 sq. J. S. Brewer Letters and Papers ... of . Henry VIII. III, p. 373. 1572. Das Bistum Erlau im Werte von 12000 Dukaten jährlich erhielt der Vizekanzler Medici.

z) Vgl. Hergenröther im VIII. Bd. v. Hefele's Konziliengesch. S. 596, 616 ff., 641, 702, 726. Grabschrift bei L. Schrader, Monum. Ital. Helmstädt 1592, fol. $146^{\prime}$ '.

s) ADB: De solenni festu Paschae ad reteres collatio (Lauchert).

4) J. Ziegleri . . contra heresim Valdensium 11. T. Leipzig 1512. 
Unreife der Schrift einmal mit dem Drängen des Bischofs von Olmutz, Stanislaus Turzo, der ja wie sein Brader Johann V., Bischof von Breslau, zu den Gönnern des Erasmus gehörte $;^{1}$ ) aber er verurteilt zugleich rückhaltlos die abstoßende Härte seiner zu einer Gewinnung des Gegners wenig geeigneten Polemik. Wie weit er sich den Bestrebungen Luthers damals schon genäbert hatte, geht ja einmal aus seiner rein auf das Schriftwort gegrundeten Verteidigung der Abendmahlsfeier unter beiderlei Gestalt hervor, noch mehr aus seinen auf Klärung und Verbreitung der evangelischen Grundlagen des Glanbens") gerichteten Bemthhungen; dagegen hält er sich mit seiner öffentlichen Polemik gegen die kirchliche Reaktion noch in den fur den Erasmianer bezeichnenden Grenzen: er mißbilligt zwar in vertraulichem, zu sofortigem Verbrennen bestimmten Briefe die Schmähang der Person Lathers; als literarischer Schildknappe aber springt er nur dem ihm seit vielen Jahren befreundeten Oberhaupte der Humanistengemeinde bei.

Und somit begnigt er sich einem Eck, dem Widersacher Lathers, gegenuber, bei vorsichtiger Verschweigung des Namens mit einer drastischen Schilderung seines Auftretens im Rom, wo der große Meister akademischer Redeturniere, der einst in Wien and in Bologna im Sold und Auftrag der Fugger das den kirchlichen Satzungen widerstreitende Recht des Bankiers auf Verzinsung seiner Darlehen verteidigt hatte, nun an der römischen Universität, der Sapienza, zu wiederholten Malen sein Licht leuchten ließ.

Eck war am 21. Oktober 1521 zum zweitenmal nach Rom gereist, um uber die Vollziehang der Bannballe zu berichten und die kirchenpolitischen Forderungen seiner Herzöge zn vertreten. ${ }^{\text {) }}$ ( DaB das anmaßende, geränschrolle Wesen

1) Erasmi opp. III, col. 668 sq. 712.

2) Er legt auch in unserm Schreiben den größten Wert anf den Plan einer Evangelienbarmonie (quatuor evangeliorum perpetaa historia), die er in der Schrift gegen Stunica ausführlich ankündigrt.

3) Th. Wiedemann, Dr. Joh. Eck, Regensburg 1865, S. 184 ff. S. Riezler, Gesch. Baierns. IV. Bd. (Gotha 1899), S. 77 f. C. v, Höfler, Papst Adrian VI., Wien 1880, S. 326: Erlaß Leos X. v. 15. Nov. 1521. Yach nnserm Briefe war Eck am 16. Februar nach Vereitelung seiner Bemühungen durch den Tod des Papstes schon wieder abgereist; er traf noch im Februar in Ingolstadt ein. 
Ecks ibbrigens auch weiteren Kreisen in Rom wenig zusagte. ersieht man aus der Mlitteilung Melchiors von Watt: Eck werde hier durch sein Gebahren (quod ad mores spectat) zum allgremeinen Gelächter. ${ }^{1}$ )

Dr. Eck also war es. der bei seiner Ankunft in Rom der $\mathrm{K}$ urie berichtete (ad nos retulit), dab ,isthic", in seiner Heimat, d. h. wohl in Deutsehland uberhaupt, nicht in Baiern, schon ein paar hundert Menschen das Abendmahl unter beiderlei Gestalt genommen hätten, und Ziegler schildert nun spöttisch das Entsetzen der Kurialen über dieses von Eck nachdrücklich charakterisierte Verbrechen. Dab hier keineswegs an eine lirbreitung lutherischer Lehren in Rom \%u denken ist, geht auch aus der nur zu richtigen Bemerkang hervor. die er 156 gegen Vadian²) macht: "uber die deutschen Verhältnisse herrscht in Italien die größte Unwissenheit; wenige nur und zwar nur die Kirchenfursten erfahren etwas, was ihuen einige ihrer geschworenen Parteiyainger von dort (istinc) berichten, was jedoch dem Volke wie ein Staatsgeheimnis verhohlen wird. Nur durch die lnwissenheit glauben sie ihrer Herrsehaft uber die Kirche sicher zu sein, die ihnen mit der Aufklärung des Volkes zusammenzubrechen droht."

lind so durfen wir uns gar nicht wundern, wenn bei Gelegenheit eines Gastmahls bei einem hochgestellten Kurialen von bischöflichen Range das l'rteil über Luther einfach dahin lautete, dab er ,ein Hurer und Säufer" sei; die in ihren materiellen Interessen, den Bezügen von ihren deutschen Pfründen, grfiihrdeten ,alten Kurtisanen" erklärten ihn gleichzeitig") kurzwegr fiur "den Vorliafer des Antichrists".

Dagegen stand Erasmus in diesen Kreisen anscheinend noch im vollen Genusse seines unvergleichlichen Rahmes, wenn er bei jener (ielegenheit als "die Sonne" der gelehrten Welt gefeiert wurde. Doch wagte sich der schon seit Monaten in Rom eifrig gegen Erasmus agitierende spanische Theologe Jakob Lopez Stunica (Zuñiga, $†$ 1530) auch in

1) Mitt. des St. Gall. Ver. XXV, s. 281.

$\therefore$ A. a. O. XXVIII, S. 35.

3) A. a. O. XXVII, S. 215 (Melch. v. Watt, den 16. Febr. 1521). 
dieser erlesenen Versammlang mit einer Probe seiner Inrektiven hervor, indem er offenbar auf die bekannte Stelle im Lobe'der Torheit anspielte, in der Erasmus uber die Prahlerei der Mönche mit den ÏuBerlichkeiten ihrer Regel, „eine bestimmte Anzahl Knoten an den Schuhen,.. das Gewand aus bestimmt vielen Lappen zusammengeflickt, .. die Kapaze von bestimmter Weite and bestimmtem Schuitt" sich lustig macht. Nun hatte man ja anßerhalb der vertrantesten Umgebung des Papstes und des Vizekanzlers damals offenbar noch keine Ahnung von dem infolge der Berichte Aleanders seit dem verwichenen Herbst allmählich eingetretenen Umsch w ung in derGesinnung der leitenden Persönlichkeiten gegen Erasmus, ${ }_{1}^{1}$ den der scharfblickende, unerbittliche Gegner nunmehr schon durch seine nicht miBzuverstehenden Drohungen and Verdächtigungen, librigens ganz nach dem Wortlant seiner Instruktion, ${ }^{2}$ ) da er ihn nicht gut dem Scheiterhaufen uberliefern konnte, wenigstens aus seiner Heimat und von der Universität Löwen vertrieben hatte. Wenn nun Ziegler berichtet, daB bei einer zweiten Begegnung mit Stunica im November der Freand und Korrespondent des Erasmus, Paul Bombasio, den boshaften Angriffen des Spaniers ebenso wie kurz vorber im Beisein des Papstes, dem jener seine Schrift mit dem gegen Erasmus erhobenen Vorwurf der Ketzerei uberreicht hatte, mit einer wohldurchdachten Verteidigang entgegengetreten sei, so war es ja doch gerade dieser Bombasio, der Sekretär des Grobpönitentiars, des Kardinals Lorenzo Pucei, durch den der argwöhnisch gewordene Papst im Sommer einen bedeutsamen Wink an Erasmus hatte weitergeben lassen. ${ }^{3}$ ) Und auch jener bischöfliche Gastgeber, der die hitzigen Ausfälle Stanicas angehört und dabei den Erasmus für einen ganz gefährlichen Menschen erklärt hatte, gehörte offenbar zu den Eingeweihten. Man wunschte jedoch einen offenen Bruch mit dem großen Gelehrten zu vermeiden, den man ganz im stillen, jedoch

1) Vgl. meine „Anfänge der Gegenreformation in den Niederlanden" (Schr. d. V. f. R.-G., Halle 1903, 04) I, S. 88 f. und II. Kap. V: die Verdrängung des Er. ans den Niederlanden, S. $38 \mathrm{ff}$.

*) P. Balan, Monum. ref. Luth., Regensburg 1884, p. 10.

3) Anfänge der Gegenref. I, S. 89 f. 109. II, 46. 
mit allem Ernst zu kirchlichem Wohlrerhalten zurtuckzufuhren entschlossen war. So viel war auch dem dentschen Freunde des Erasmus klar geworden, daß dessen Gegner dem Siege nahe gewesen waren, und Bombasio hatte ihm, als Beweis fiir die schon halb gelungene Einschuchterung des Erasmus, dessen an ihn selbst gerichteten Brief rom 23. September gezeigt, in dem Erasmus sich am die ihm von Aleander boshaft verweigerte Erlaubnis des Papstes bewarb, Lathers Schriften, die za widerlegen er beständig gedrängt warde, wenigstens auch lesen zu durfen; ${ }^{1}$ ) Bombasio sollte ihm ein entsprechendes Breve erwirken; er werde sich dem Papste dankbar erweisen, wenn er nar noch drei bis vier Jahre lebe: ein Versprechen, das Ziegler mit skeptischem Lächeln las.

Während nun Ziegler in wachsender Erkenntnis der Gefährlichkeit and Verbissenheit Stunicas den Plan zo seiner $V$ 'erteidigungsschrift, dem libellus adversus Jacobi Stunicae maledicentiam entwarf, versuchte anch schon ein Landsmann des Spaniers, der in Löwen sich mit Erasmas besprochen hatte und dessen ganze Lebensfuhrung zu beurteilen wisse, brieflich auf Stunica einzuwirken und ihn durch eine glänzende Schilderung der-Verdienste des Rotterdamers von seinem verbrecherischen Vorhaben abzubringen; es kann dies kein anderer gewesen sein als $\mathrm{Ludovic} o \mathrm{Vives}$, wenn nicht der wissenschaftlich bedeutendste, so gewiB der sittlich tüchtigste unter den niederländischen Freunden des Erasmus. $\left.{ }^{2}\right)$

Inzwischen aber war schon ein anderer Spanier in Rom selbst dem Stunica zur seite getreten, um ihn in seiner Agitation, wie etwa bei Besuchen in den Bücherläden, wo er die literarisch Gebildeten gegen Erasmus einzunehmen beflissen war, doch auch mit der Feder zu unterstutzen: es war dies Dr. Sanchez Carranza, ein Theologe ans Alcala de Henares (Complatum).

Stunica selbst ist schon im Fruhjahr 1521 nach Rom gekommen, da Melchior von Watt schon am 22. April seinem Bruder meldet, daß ein neuli $\mathrm{ch}$ in Rom eingetroffener Spanier - in einer Nachschrift $v 0 m$ 24.4. spricht er ausdrucklich

1) A. a. O. II, S. 49 . Er. opp. III, col. 665 B; so erklärt sich auch der nächste Satz unseres Schreibens: Vidi triennium etc.

$\left.{ }^{2}\right)$ Anfänge I, 70. I, 56. 90. 
„de $\mathrm{J}$ a c ob o L a pide* - in einer gewandten. schon vor einigen Wochen gedruckten Streitschrift gasz gewaltig gegen die erasmische Ü̈bersetzung des Neuen Testaments und die dazu gehörigen Annotationes losziebe ${ }^{\mathrm{I}}$ ), und zwar zur Verteidigung der Vulgata. Auch dem jungen Schweizer war dabei die theologische Unfruchtbarkeit und literarische Schwerfälligkeit der Italiener aufgefallen. die derartig auf die Unterstutzung durch spanische Hilfskräfte angewiesen waren. ${ }^{2}$ )

Die Schilderang nun, die unser Ziegler von den Treibereien der beiden Spanier gegen Erasmus entwirft. wird ergänzt durch die Übersicht uber ihre Streitschriften und die ihnen entgegengesetzten eigenen Publikationen des Erasmus, von denen dieser in dem periodisch veröffentlichten und nach Bedarf vervollständigten „Katalog" seiner bei Johann Froben in Basel erschienenen Werke berichtet: da erzählt er in dem am 30. Januar 1523 abgeschlossenen Drucke, wie Stunica nach dem mit seiner ersten Schrift in Rom gefeierten Triumphe ein zweites Buch, die Blasphemiae et impietates Erasmi vollendet, dessen Drack jedoch von den Kardinälen (während der Sedisvakanz und der Abwesenheit des neugewählten Papstes) verboten wurde; mit Hilfe der Mönche aber wurde nicht nur der Druck, sondern auch, trotz erneuten Verbots, die Veröffentlichung vorgenommen; dann folgten noch drei Schriften, die er jetzt mit päpstlicher Ermächtigung herauszugeben wunscht; Carranza aber beschränkte sich darauf ihm in drei Punkten beizuspringen and die Ketzerei des Erasmus za erweisen; das Schlimmste sei, dab sich in Rom Leute fänden, die derartiges kauften und also doch auch wohl läsen, während man dort kaum einmal die Schriften der um die christliche Religion hochverdienten Kirehenväter zu kaufen Gelegenheit habe. ${ }^{3}$ )

1) Mitteil. d. Ver. v. St. Gallon XXV, S. 361 and XXVII, S. 218.

2) Italis aliena est haec Germanorum in literis experientia et amoenitas (XXV, 371), was er nur im Zusammenhange seines Berichts über die gegen Luther erschienenen Streitschriften äußern konnte. Ende Jnli 1521 war Melchior v. W. in die Dienste eines vornehmen spanischen Familiaren oder Cavaliers (scutifer, écuyer) des Kardinals Lorenzo Pucci getreten. l. c. S. 382.

3) Catalogus omnium Erasmi Roterodami lucubrationum, ipso 
Gerade als dieses Heftchen in Druck war, kam ans Rom, wie der gelehrte Buchdrucker Joh. Froben in seiner Vorrede berichtet, die sichrift Zieglers gegen Stuniça in Basel an (Dez. 1522) nud wurde bald darauf als hochwillkommen und zeitgenäb mit empfehlendem Hinweis auf das com Verfasser zu erwartende Evangelienbuch abgedruckt und zugleich mit den vorangestellten Arbeiten des Erasmus im April veröffentlicht; Ziegler hatte seinen Vorsatz also im Laufe des Jahres 1522 ausgefubrt; er bezeichnet als positiven Zweck seines Dialogs mit Stunica neben der Verteidigung der erasmischen Übersetzang des Neuen Testaments die Vorbereitung jener "Konsonanz" der Evangelien, indem er sich auf die Arbeiten des Ammonius ron Alexandria, des Hieronymus und Augustinus und letzthin auf den entsprechenden Plan Johann Gersons beruft.

Begleitet war diese Sendung von einem zweiten reichhaltigen und geistreichen Briefe, dessen Inhalt wir ans der Antwort des Erasmus vom 22 . Dezember 1522 ${ }^{1}$ ) leider nur zum kleinsten Teile rekonstruieren können, da der Empfänger die wiederholte Mahnung, die er inbetreff des ersten Schreibens trotz der hier gegebenen Versicherung außer Acht gelassen hatte, diesmal beherzigt haben dürfte; man sieht auch warum: Ziegler hatte sich sehr ungünstig über Hadrian VI. geäußert, und Erasmus versicherte ihm nun unter vorsichtiger Andeutung

autore. Cum aliis nonuullis. Im schlusse: Basileae, tertio Cal. Febr. MDXXiij. Es folgt neben einem Briefe an die Löwener Theologen das yroße Verteidigungsschreiben des Erasmus an Marcus Laurinus (rom 1. Febr. 1523) und danu mit besonderem prächtigen Titelblatt der "Libellus Jacobi Ziegleri Landaui Bauari adrersus Jacobi Stanicae maledicentiam, pro Genmania. Auf der letzten Seite ein stattliches Druckerzeichen und: Basileae in aedibus Joanuis Frobenii, Mense Aprili. An. MDXXIII. - Der Bericht iiber die Angriffe der beiden Spanier wird dann ergänzt und bis auf das vou Clemens VII. an Stunica erlassene Verbot fortgeführt in dem ${ }^{\text {Catalogas noras }}{ }^{\star}$ rom „September $1524 "$, wie auf dem Titel und am Schlusse vom Drucker vermerkt wird, während der Verfasser das frïhere Datum rom 30. Januar 1523 an Ende versehentlich hat stehen lassen. Wir erfahren hier, daß schon Leo X. die Herausgabe der zweiten Schrift die erste war noch in Aleala gedruckt - verboten batte. Exemplare der Bresl. Univ.-Bibl.

1) Er. opp. W, col. $738 \mathrm{sc}$. 
der P'rson des Pontifex Maximus, daß der Freund hierin zu schwarz sehe; wenigstens was ihn selbst angehe, durfe er sich rom Papste eines Besseren versehen. da dieser es abgelehnt habe, nach den gehässigen Wüuschen seiner Feinde gegen ihn einzuschreiten, ron denen er den gefährlichsten, der im geheimen gegen ihn intriguiere und die andern vorzuschieben pflege, Hieronymus Aleander, nicht nambaft zu machen wagt (col. $739 \mathrm{~A}$ ).

Fur die ubersandte Verteidigungsschrift dankt er ihm mit den wärmsten Worten und in wohlverdienter Anerkennung seines schriftstellerisehen Talents; nur bedanert $\bullet r$, daß Ziegler so viel Fleiß und Kunst an einen so schäbigen Gegner verschwendet habe, obwohl ihn Erasmus in einem Briefe, der sich mit dem ersten Schreiben Zieglers gekreuzt hatte, freundschaftlich davor gewarnt hatte, in dieses Wespennest zu greifen; dieser hatte die ÄuBerung so verstanden, als ob ihn Erasmus um Rat frage, ob man dem Stunica etwas entgegnen solle; er mitsse wohl die Apologien des Erasmus gegen die beiden Spanier nicht gelesen haben. Den von dem Freunde nicht ausdrücklich erbetenen Abdruck des "Libellus" hat sich Froben natürlich nicht entgehen lassen; doch bittet ihn Erasmus das angekundigte Werk de contextu IV evangeliorum nicht mit Stunicas Namen zn belasten.

Ziegler hatte ibn ferner $\% u$ uberreden versucht, seinen Wohnsitz zu verlegen; Erasmus aber erklärt es rund heraus für ganz aussichtslos, ihm die Vorteile eines solchen Schrittes auszumalen; Alter und Kränklicbkeit waren jedoch nicht die eigentlichen Gründe fur die Ablehnung eines Projekts, das er den kurialen Machthabern gegenuber seit Jahren schon als einen Lieblingsplan vorzubereiten sich den Anschein gegeben hatte; vielmehr wuBte er zum mindesten seit dem Sommer 1521 genau und gab es auch Ziegler gegenuber zu verstehen, was er von der Aufrichtigkeit der kurialen Kreise halte (quanta sit isthic quorundam integritas!); er habe dies ans der Daldung der Angriffe Stunicas deutlich genug ersehen und wisse anch genau, auf wessen Antrieb (quorum instinctu) dieser vorgegangen sei. Nun war einmal Aleander in diese 
Dinge gründlich eingeweiht: ${ }^{1}$ ) einen andern hohen Gönner des Spaniers hatte ihm Ziegler soeben nambaft gemacht: es war dessen Landsmann, der greise Kardinal tit. S. Crucis, Bernbardin Carrajal, aus dessen Widerspruch gegen den antikonziliaren Charakter der Bulle Exsurge Erasmus allzu optimistische Folgerungen gezogen hatte. ${ }^{2}$ ) Dieser hatte den verhabten Gegner als Familiaren in seine L'mgebung aufgenommen und Erasmus fand nun, dab die beiden einander wert seien. Indessen war das einstige Oberhaupt des Konzils von Pisa seit dem 16. Dezember nicht mehr unter den Lebenden. ${ }^{3}$ )

Erasmus versäumte nun nicht, dem alten Freunde, der ihm so eifrig beigesprungen war, auch durch eine earenrolle Erwähnung in einer gleichzeitigen Kundgebung*) zu danken, indem er seinem literarischen Talente eine glänzende Zukunft prophezeite. Doch durfte diese Freundschaft schon in den nächsten Jahren, als Ziegler den deutschen Landsknechtsführer Frundsberg als Sekretär auf dem Zuge gegen Rom begleitete und dann auch in briefliche Verbindung mit den Wittenbergern trat, sich aufgelöst haben. Wir besitzen anscheinend kein Zengnis für eine Fortsetzung der Beziehungen zwischen beiden Männern, aber vielleicht hat Erasmus auch spïtere Briefe seinem Versprechen gemäß vernichtet.

Fur den geschichtlichen Wert der Historia Clementis VII. aber, wie der noch ungedruckten, doch schon von Ranke für die Schilderung des Sacco di Roma benutzten „Acta Paparum

1) Anfänge d. Gegenref. II, 48, 45.

*) $\mathrm{Zu}$ Lnthers röm. Prozeß, ZKG. XXV, 120 124. $512 \mathrm{f}$. Über eine merkwürdige auf Erasmus zurückzuführende literarische Aufmerksamkeit seiner Antwerpener Freunde für den Kardinal C. aus d. J. 1520/21, vgl. meine im Repertorium für Kunstwissenschaft Bd. XXVIII. Berlin 1905 erscheinende Untersuchung über ${ }_{n}$ Albrecht Dürer, Sebastian Brant und Konrad Peutinger in Antwerpen im Sommer $1520^{4}$.

3) Dictionnaire des Cardinaux, Paris 1857, s. r. C.

1) Er. opp. III, col. 738. - Unter seinen Freanden in Ron zählt er neben Paul Bombasio und den bekannten Deutschen Joh. Goritz und Georg sauermann ins Breslau auch auf den Landarus olim futurus inter scriptores nobilis. l. c. col. $754 \mathrm{~A}$. 
Urbis Romae" hat sich aus vorstehender Untersuchung zum mindesten ergeben, dab eine eingehende Prufung und Verwertung der in so intimem Verkebr mit den leitenden Kreisen gesammelten Beobachtungen sich wobl verlohnen dürite.

\section{Jakob Ziegler an Erasmus.}

Rom, d. 16. Febr. 1522. Bresl.Stadt-Bibl. Hs. R. 254, ur.167, p.456 463.

S. P. Dicit. Veteris amici tai et te amare incipientis, primam quam tu eruditis et iuxta piis studiis innotuisti, seram epistolam ambitiosis, id quod multi faciunt scribentes ad te. salutationibus auspicari forsan conveniebat; sed facit ingenii vel inopia vel rusticitas, ut abstineam ab eiusmodi exordio. Debebam deinde excusare dissimulationem ad te meam maltorum annorum, verum neque hic volo laborare, quin qui tacui et pro viribus favi tamen; tectus favor debebit tibi isto dulcior videri, quoniam ab isto exemplum capis in me, quo docearis, reliquisse Deum malta milia virorum, qui tibi devoti sunt et genu suum ante Baal non curvarerunt.

Eas literas te accoepturum puto Basile ae, quod equidem praecapiam certus, quia isthic exprimes aliquid ad publicam rei christiauae utilitatem. Expecto autem ex tua censura Aurelium Angustinam, quem nimis deformem habemus, quoniam hunc nescio qui Angli et Maiorones indocti suis neniis et feritatibus misere dehonestarunt. Quare A morbachii senis iudicium vehementer desidero. Tantum ergo auctorem videre cupio repurgatum et suae ipsius restitutum dignitati. Nam quare tua in Paulum commentaria tocies quamvis promissa et ostensa nobis, hoc tempore non sperem, tam intelligo coniecturis rerum, quam ta ex animo nosti. Posthamum suscipiemus partum istum videlicet; cum hic tam pluribus aliis rebus profecto me tangit quidam tui animi sensus.

Apud Cyprianum etc. (cf. opp. Erasmi Ш, col. 1699 sq.)

[p. 457] Venerunt Romam propugnatores duo Pontificiae stationis, ${ }^{1}$ ) qui hoc anctumno uno proposito eam in gradu retineant, a quo detradere quidam moliuntar, et ut premia et sacerdotia ${ }^{2}$ ) propterea referant, atque adeo alieni capitis delatione beati evadant. Is, quod nosti, congressionibus suis scholasticis testatum olim foecit, sophistam esse se unum invictum. Saepe his etiam diebus conflixit in Sapientia Romana, et fuit vehementia hominis, caput iactatum, latus molle, manus loquens et causam partiens in digitos atque in

1) Dr. Joh. Eck und Stunica.

2) Ziegler wußte, daß Eck eine günstige Entscheidung des Papstes in seinem ProzeB um die Pfarre von St. Moritz in Ingolstadt zu erwirken hoffte. 
his os apertum nimis stupori omnibus. Erro nihil non speramus nos obtinere per illun. qui theologicae definitionis titulo maxime paradoxon ehristianae philosophiac dogma semel et publice obtinere visus est. ius esse mensariis foenoris accipiendi. Hune festivi quidam delicias aint esse nominis fiermani et remiserunt robis. Equidem et ego (nisi quia falsum est: crimine ab uno disce omnes) agnoscerem vel invitus opprobrium gentile.

Alteri diversa ratio est: $|p .458|$ huius incessus, sermo, rultus totusque habitus compositus ad quandam modestiam videtur, praefert etiam hypocrisin in media causa, qualem non temere reperias apud magnos auctores; longe abesse putatur ab assentatione et consectatione lueri ad rerum dominos, longe $a b$ odii et offensionis affectu in adversarium. unde rebus fides levior baberetur, sapere autem, dicere et scribere omuia, quae veritatis puram defensionem, regulam apostolicam et veterum tractatorum referant sententiam et fidem. His artibus instructus libellos Romam itentidem submisit, praesidium moribus et opinionibus nostris adferens, quibus hoc seculo summus rei christianae cardo nolens volensque versatur. Hoc consilio desiderium sui longe ante foecit. isto etiam insignius, quod rocantibus non semel dominis non properarit, scilicet hoc venerabilior occursurus, boc ampliores provocaturus fortunas, quo se graviorem, quo sui copiam minus impetrabilem ostendisset. Nec eum sua prudentia fefellit; fortunam a porta in sinum fere elicuerat, sed humanarum rerum casus illi omnes spes evertit, namque sub ipsius adventu Leo decimus gravem animam efflavit.

Sed audias, oro, ab uno exemplo, quae ista sint prava dogmata, quae hi ferre non possunt. Isthic trecenti Christiani synaxin sub utraque specie celebrarant. Id is ) ad nos retulit et tanquam sacrilegio perpetrato exaggeravit. Nos ut rem funestam excoepimus, exhorruimus, facinus omni calamo et censura damnandum duximus, pato quia contra maiorum morem, contra manifestam scripturaram sententiam foecerunt: Amen, amen, dico vobis, nisi ederitis carnem filii hominis et biberitis eius sanguinem, non habebitis vitam in vobis. Atque haec sententia quater repetita; et alio loco: Accoepit Jesus panem et, cum aegisset gracias, fregit deditque discipulis et ait: accipite. comedite, hoc est corpus meum. Et accoepto poculo, graciis actis dedit illis dicens: bibite ex hoc omnes. Et Paulus: probet autem homo seipsum et sic de pane edat et de poculo bibat, atque et hoc loco idem ritus tocies commemoratus. Sic maniti descendunt in certamen assertores publici moris et male olim

1) Dr. Joh. Eck. 
recoeptarum opiuionum. $O$ nuuquam. qui sancta legit et versat. sed qui oculum simplicem, humilem spiritum. mentem doctam a deo exhibet. bene pius est.

leni ego Romam annosuperiori ipsodie Calendarum Martii, evocatus ex Lngaria per Leonem Decimum cognitionem instituente Hippoly to Estensi cardinali, qui episcopatum in Lngaria babuit omnium opulentissimum; quo loco is mea studia cognoverat et amaverat. Salutavi Pontificem ab opere quodam meo in hereticos pighardos, inter quos antea fueram triennio conversatus. Hoc alias mihi exciderit. quoniam celeritate gratificari volebam episcopo Olomucensi, qui idem efflagitabat; ergo huic ut festinato operi multa acciderant non ferenda. Plus materiae quam corporis instus modus postulabat; dispositio multis partibus enormis, calor in contentionibus maior, quam ferret christiana aut denique modestia aliqua civilis.

Hoc vitiom veniebat mihi, quod experimento [p. 459] sciebam, quantum oratio a sententia et vita illorum dissidebat. Quod tamen excusari non potest in argumento, quo studeas adversarium in tuas deducere partes, videlicet, qui causae concoedere poterat, enm acerbitate exasperare prius, ut memoria doloris obsistat ${ }^{1}$ ) nec possit cum illo. qui contumeliam foecit, aliquid sentire commune.

Romae ubi fui, invitamur ego et perduellis tuas ${ }^{2}$ ) apud magni nominis hominem ex episcoporum numero, ubi, ut semota mensa fuit, productus est in coronam Lutherus et varie agitatus; summa sententia erat scortatorem eum esse et compotorem, qualibus viciis fere laborarent Germani. Secundum ipsnm to non taa persoua prodiisti. Risa amaro excipiebat perduellis, quod solem plaerique te salutarent, qui tua in Christo pia studia agnoscant. Lecta deinde est ipsius vel epistola vel in aliud opus praefatio quaedam, in qua in invidiam adducebantur loci, quibus ta monaciscos, preculas avidas, ancisas cucullorum oras, rugas palliorum adnumeratas et omnia secoessionum in ecclesia symbola insectaris. Et senex ille alioqui non monachus, sed sacrificulus, tamen ater et inamabilis tui nominis impatientia totus ferebatur. Favebat huius candori communis hospes, quia parum aequiter forsan audiret dici in Germania illustre aliquid. Tibi etiam privatim non am ic us esset, quem clamitaret habere in cornu foenum. Videlicet Evangelium et Canonem omnem divinum vindicem viciorum olim oblitterarunt homines sensu corrupti; substituerunt antem illi theologiam quandam obnoxiam et tradiciones obsequentes suis

1) So zu lesen statt absistat.

7) Stronica. 
auctoribus. quibus legibas deinceps securi in omnia pro libidine feruntur. Et qui haec ad divina rursus decreta attrahit et expendit, cum invidiam movere dirinis libris non audeant, rindictam pro dolore in eum exercent, qui deprehensam nequitiam ad locum oportune subnotavit. Ab hoc affecta foeliciter illi occurrerat, qui nasum tuum coeno quamvis foedo ultus esset. Referebam ergo haius coronae fastidiam et stabam iniquo loco, tamen ea inter nos pace dicta, ut qui civiliter accoeptus essem. Discoessimus; perduellis quidem tuus ad munus suum, nam in Sapientia Romana stipendio oneratus Oppianum enarrabat. Hune auctorem habebat unum omnium gravissimum, a quo suas opes ostentaret Romae et piscario foro indicaturam faceret homo, qui non paucos annos in sanctis scripturis veteris ac novi testamenti hebraice, graece et latine perlegendis consumpserit et qui si quispiam alius et ipse his de rebus suo quodam iure indicium ferre possit.

Ego autem domum mean minarum plaenus et iam inde concipiens animo locum oportunum, quo malivolentiam hominis intollerabilem merito exciperem stilo. Id facturus eram a prologo in perpetuam evangeliorum quatuor historiam, quam Aurelius Augustinus opere de consensu evangelistarum ostendit modo, sed non prestitit. Dum ego in hoc consilio essem, convenimus rursus mense Novembri interfuitque coronae Paulus noster Bombasius. Hic perduellis iste tuus secundo produxit [p. 460] in fabulam reterem suam non nunc praefationem, sed iustum opus ad omnem maledicentiam et calumniam heresis accuratum, ut tu auctor unus credaris omnis perfidiae, quae sub coelo et in tartaro comminisci a deploratis mentibus et ab omni pietate desertis unquam potuit. Hoc legebat in corona, hoc paulo ante Leoni decimo porrexerat $^{1}$ ) induxeratque homines, ut male de te inciperent suspicari. Paulus Bombasius utroque loco. in corona sua praesentia apud patronum, apud Leonem meditata a pologia malivolentiae obsistebat, te et pietatem tuam magnis animis asserebat. Tamen in proclivi erat, ut pars deterior vinceret, nisi isti malivolentiae Leo decoessisset. Nosti adhuc, quid ex te voluerint; experimentum hoc erat, praesensisti et ta atque vidi a literis tuis ${ }^{2}$ ) timorem istum et putare te nec posse legere quosdam, priusquam apostolica venia admittaris. Vidi triennium istud tanquam inducias gerendi belli postulatum. Risi tacitus mecum, et dicere fere tecum mihi videbar: Multa inter labrum et poculum! Hactenus,

1) Er. an Pirkheimer, d. 28. Januar 1522: Habet Roma Stunicsm . . Is exhibuerat Leoni libellum, qui continebat sex myriadas heresium decerptas a meis lucubrationibus et ea causa periclitabar, nisi mors intercepisset Leonem alioqui non infensum Erasmo. Opp. W, col. 709.

7) Vom 23. September 15.1. 
donec putavi perduellem tuom aliquem esse in literis et consciencia christiana magnum, haec me vehementer urebant atque impatiencia actus remis et velis in tuae causae apologiam scribendam properabam, sed cum sequentibus diebus aliquocies conveniremus et is se magis in dies aperiret laevius, coepi ferre insaniam hominis, arbitratus malam fabulam mimum pessimum cito prodituram. Et nunc ille de opinione perit. Scripsit ad hunc quispiam illius gentilis, ${ }^{1}$ ) qui tecum Lovanii loquatus tuam domesticam consuetudinem omnem deprehendit, honorificas literas super tuo nomine, hoc agens, ut perduellis tuns merito virtatis tuae a scelere discoederet. Sunt hic. qui eum domi noverunt; aiunt illhic fuisse habitum atheum. Certe ego Hebionitam ipsam semper putavi. Paulus Bombasius gravi vultu foedam illi adulationem, nefariam sectandi lncri sub innocentis delatione rationem opprobravit; dixit fnnestiorem esse omni insimulata heresi Erasmiana. Elusit is increpationem risa Domitiani, non tamen suffusus padore fuit. Mihi et pluribus expostulantibus eius acerbitatis in te licentiam aliud aliis respondet: Non hoc Erasmi esse publicos mores lacessere, se freno cohibiturum Erasmi lasciviam et, quod sceleratius est, se gloriam querere ex Erasmo. Circumvolavit etiam circumforaneus homo tabernas librarias, per eas traducit et obtrudit nomen tuum inxta nolentibus et volentibus, solicitat quos potest suae vesaniae complices et reperit nunc suae farinae gentilem. ${ }^{2}$ ) Exemplaria nunc proposita sunt, quae tibi Scotinis larvis, quales tu semper exhorraisti, - ab his longe ubique declinasti, - hicceitatibus et isthicceitatibus sint occursura. Ipse etiam auctor tragoediae rarsus excutit tuam posteriorem emendationem Novi Testamenti et in ipsum Annotationes illhuc erupturus, si deo placet, at in Aethneas crates Erasmum pertrahat, [p. 461] ipse frigidus Empedocles. Non sperem, ut illius in te animi omnem furorem possim pro merito declarare. Invidia et odio tui, sui philautia est tota mente emotus. Tam ipsius personam expressam aliena oratione dabo.

Habes perduellem taum, nisi forte illi sectatas Thaidas detrahas; praeterea habes omnibus numeris militem comitum. Haec putavi te scire oportere, non ignarus oratorem ita demum dicturum causam apposite, ubi probe perspexerit adversarii habitum, consilia et mores.

Quod nisi hoc consilium foret mihi ad te, forsan excusare levitatem aegre possem, qui post tam pertinax maltoram annorum silentium ita demum scribendum putarem, cum

1) Ladorico Vives.

2) Sanctius Carranza. Im folgenden eine Verspottung der Schriften dieses Scholastikers, seiner Progymnasmata logicalia und des Antiparadoxon de ... quidditate pro Aristotele.

Archiv fur Reformationsgeschichte III. 1. 
stadia hominum pingere liberet. Ergo, quod adversarius ea ubique in se admittit, ut iam nihil sibi animi et conscientiae christianae integrum, nihil civilis honestatis inculpatum in se relinquat, censeo optimum fieri posthac, Erasme optime, ne te quoquo modo in turbam demittas, id est. dum respondes, recoedas longissime a filo. quem ipse consectandum proposuit. Sed orationem revoces ad genus, hic studium nefarium externorum, traductam ab his simplicitatem, pietatem maiestatemque germanam. vexatam patientiam tu heroici animi renerabili quadam amplitudine et gravitate toti germano homini exponas. Quorsum evasura sit actio intelligis, nec aliud remedium tandem valiturum est. Quid, tu enim his brachiis, hoc exili corpore diu et frustra oblucteris omn flumine longe lateque se ubique in nos effundente? Domi tuae consistas, a too limite aggerem vel brevem opponas oportet, ad quem aquarum moles subsidat et impetus elidatur. Boni consulas, oro Erasme; nihil ego foelicissimae tuae Minervae praescribo; tamen sit hoc mihi concoessum dicere; propterea quod tu, cum meam erga te devotionem boc sermonis genere intelligas, me ames et mei candoris testimonium apud te perpetuo confirmes. Multi tua consuetudine pene ociose fiuuntur, quibus has opportunitates concoessas esse pene invideo, cum illorum etiam, qui tuis consiliis in seriis rebus utuntar, non temere unas reperiatar in sescentis, cui magis quam mihi fuerit opus hoc et pluribus negociis gravibus et necessariis tua uti praesenti conversatione. A pologiam ego meditor pro tua innocentia; ea a limine multum progressa est, sed quando tandem suum spacium perficiat, quando ad te perveniat, nescio. Ibidem ego in multa vocor [per perduellem istum, quae si pro rei dignitate sire et indignitate persequar, habeam periculum ubi permanarint. $\mathrm{Si}$ transeam et relinquam, deseruerim stationem. Libellum deinde concipio accidentium in perpetuam evangeliorum quatuor historiam, quo eadem necessitate concludor, propter quae dolet mihi tam longe excludi a vobis, a te, a Frobenio nostro, a Germania libera. Sed et has literas per christianam nostram necessitudinem ita suscipias [p. 46\%] oro. ut tibi et statim valcano communiter missas intelligas. ${ }^{1}$ ) $\mathrm{Tu}$ si quid tuarum

1) Er. an Z., 22. Dez. 1522: De epistolis tuis, quod mones, hactenus a me curatum est et in posterum curabitur.

Furr das Eintreffen Carranzas in Rom trage ich noch eine von mir in Rom gefundene Notiz nach: Am 16. Nov. 1521 gestattet Leo X. dem Sanctius Carranza de Miranda und vier andern canonici eccl. SS. Justiet Pastoris oppidi de Alcala de Henares Tollet. dioc. etc., qui ... in artibus et the ilogia magistri seu licentiati etc. cathedram seu cathedras in theologia vel artibus in universitate opp. A. regitis, das Brevier nach römischer Art zı beten. Am 23. August erlaubt Leo dem Joh. Carranza, laicus coningatus aus Carthagena in den Johanniterorden einzutreten. (Leonis X. brevia, Arm. XXXIX, 34 f., 73, 79.) 
missurus ad me es, id deponi facias apud Paulam Bombasium, meum magnum amicam, si tamen hospicium [neu]m minus patabitur obvium. Ea autem est domus Ungaroram Romae apud [templ]am divi Petri in Vaticano. Vale, Erasme optime. Die XVI. Februarii anno salutis MDXXII.

Jacobus Zieglerus Landavus Bavarus.

[p. 463] Venerabili Domino Desiderio Erasmo Roterodamo praeceptori et amico suo inprimis colendo. Basileae [ae]des Frobenii. 